\title{
Thrombocytopenia in hypertensive disorders of pregnancy
}

\author{
Rupakala B. M., Ritika Gupta*, Vishma H. Shetty, Sailakshmi M. P. A.
}

Department of Obstetrics and Gynecology, Rajarajeswari Medical College and Hospital, Bangalore, Karnataka, India

Received: 03 January 2018

Accepted: 24 January 2018

\section{*Correspondence:}

Dr. Ritika Gupta,

E-mail: ritikagupta1602@gmail.com

Copyright: ( $\odot$ the author(s), publisher and licensee Medip Academy. This is an open-access article distributed under the terms of the Creative Commons Attribution Non-Commercial License, which permits unrestricted non-commercial use, distribution, and reproduction in any medium, provided the original work is properly cited.

\section{ABSTRACT}

Background: Thrombocytopenia is defined as a platelet count of less than $150 \times 10^{3} \mu 1$. It is commonly diagnosed and has attracted more interest from researchers during recent years, especially in Hypertensive disorders of pregnancy. This study was done to estimate the incidence of thrombocytopenia in pregnant women diagnosed with hypertensive disorders of pregnancy and to correlate its severity with the degree of thrombocytopenia.

Methods: In the study 150 women admitted in the OBG Department at Rajarajeswari Medical College and Hospital, Bengaluru during August 2015-August 2016 were included. Hypertensive disorders of pregnancy cases were classified into: Gestational hypertension, mild preeclampsia, severe preeclampsia, haemolysis, elevated liver enzyme levels, and low platelet levels (HELLP) syndrome and eclampsia. The incidence and severity of thrombocytopenia along with maternal and foetal complications encountered in the five groups were analysed. Data were arranged in Microsoft Excel version 2010, and statistically analysed by SPSS version 23.

Results: Preeclampsia- mild $(29.25 \%)$ and severe $(22.5 \%)$, accounted for most of the cases followed by eclampsia (3\%) and gestational HTN (1.5\%). Among these hypertensive patients, mild thrombocytopenia was noted in 60 cases (40\%), moderate thrombocytopenia 48 (32\%), severe thrombocytopenia $12(8 \%)$, and normal platelet counts 30 $(20 \%)$ were noted. Poor maternal outcome was seen $10.67 \%$ cases due to HELLP syndrome and postpartum haemorrhage. Poor foetal outcome was seen in $16 \%$ cases due to intrauterine growth restriction and perinatal mortality.

Conclusions: Hypertensive disorders of pregnancy is recognized as a major cause of gestational thrombocytopenia. Careful follow up during and after pregnancy is recommended.

Keywords: Eclampsia, Hypertensive disorders of pregnancy, Preeclampsia, Thrombocytopenia

\section{INTRODUCTION}

Hypertensive disorders of pregnancy are defined as hypertension that occurs in pregnancy for the first time after 20 weeks of gestation, disappearing following delivery of the baby. ${ }^{1}$ It is the most common medical disorder of pregnancy, that leads to a complicated multiorgan failure in the mother. It is one of the most common causes of both maternal and neonatal morbidity. ${ }^{2}$

It is a global problem and complicates approximately 10$17 \%$ of pregnancies. The incidence of PIH in India ranges from $5 \%$ to $15 \% .^{3}$ Hemorrhage occupies an important factor in the etiology of maternal mortality and therefore, remains a major problem. ${ }^{4}$

Currently, there is no screening test that would help in identifying which pregnancy will be associated with PIH or assess its severity. ${ }^{5}$ A variety of hematological abnormalities may occur in women with PIH of which thrombocytopenia is the most common..$^{5-8}$

Platelets are produced in the bone marrow and remain in blood for about 2 weeks before they are destroyed in the 
reticuloendothelial system. The normal platelets count ranges between 150 and $450 \times 10^{3} \mu 1$ which is also the same as that is mostly recorded during the normal pregnancy. ${ }^{9,10}$

Gestational thrombocytopenia (GT)-thrombocytopenia during pregnancy (PIT)-occurs in late gestation, and its frequency increases during the last few weeks of gestation, and it occurs also during the last few weeks of the second trimester. PIT is commonly mild $\left(>100 \times 10^{3} \mu \mathrm{l}\right)$, and resolves usually completely after delivery; however, severe thrombocytopenia $\left(<70 \times 10^{3} \mu 1.\right)$ rarely occurs. ${ }^{11}$ Several studies have reported fetal and/or neonatal thrombocytopenia in 4$13 \%$ GT's mothers. ${ }^{12}$

During pregnancy, fluid retention occurs because of sodium and water retention under estrogen and progesterone hormone effects, leading to hemodilution. This leads to lower hematocrit (dilution or pseudothrombocytopenia). Burrows et al reported that the frequency of PIT was 5\%, while Kaplan et al. ${ }^{13,14}$ observed PIT in about $7 \%$ of pregnancies. This increase was claimed to be related to previously acquired or inherited diseases or pregnancy related complications such as preeclampsia, sepsis, or pregnancy-induced disseminated intravascular coagulation. ${ }^{14}$

The underlying cause of GT cannot usually be identified in about $75 \%$ of the cases. In such cases, GT is generally assumed to be secondary to increased platelet consumption within the placental circulation and/or to hormonal inhibition of megakaryocytopoiesis. This type of thrombocytopenia is known as asymptomatic thrombocytopenia; it, however, does not cause any clinical adverse effects in the mother or the baby. ${ }^{13,14}$ Asymptomatic thrombocytopenia is reported in $5 \%$ of normal pregnancy at preterm, whereas about $15 \%$ of pregnant women develop severe thrombocytopenia if they develop preeclampsia during the last term. ${ }^{13}$ Thrombocytopenia occurs more and slightly severer in twin pregnancy than in singleton pregnancy. ${ }^{15}$

The criteria to diagnose gestational thrombocytopenia are as follows: platelets count is normal outside pregnancy, occurs late in gestation, no fetal/neonatal thrombocytopenia and complete recovery with normal platelets count, and function after delivery. ${ }^{11}$ GT is considered a benign condition during pregnancy, and it does not require greater intensive care than routine obstetrical care. ${ }^{12,16}$

Thrombocytopenia can be due to idiopathic thrombocytopenic purpura (ITP) which is caused mostly because of the formation of autoimmune antibodies. Most of GT manifestations are similar to ITP. Letsky et al reported that differentiating ITP from gestational thrombocytopenia by antibodies immunoassays is difficult, while at least one or two of ITP-induced antibodies are present in PIT. ${ }^{16,17}$ GT is usually not severe and has not significant effect on neonatal thrombocyte count. On the contrary, ITP is usually severe during pregnancy, but it improves after delivery in mothers; and severe thrombocytopenia may occur in about $5-10 \%$ of offspring of the affected mothers. ${ }^{18,19}$

Preeclampsia prevalence is variable, the estimated incidence is $5-10 \%$ of all pregnancies, with a higher incidence in the first pregnancy especially in women aged less than 20 years. ${ }^{19}$ The frequency and severity of thrombocytopenia increase with the severity of preeclampsia, and are greater in patients with the HELLP syndrome or in those who have a full-blown eclampsia with disseminated intravascular coagulation. ${ }^{20}$ Mangann et al reported that overall incidence of thrombocytopenia in pregnancy is $8 \% .{ }^{21}$ PIT incidence drops to $5.1 \%$, when obstetric or medical conditions are excluded, and almost three-fourths of all cases are due to PIT.

A variety of hematological abnormalities may occur in women with hypertensive disorders of pregnancy of which thrombocytopenia is the most common. ${ }^{5-8}$ Thrombocytopenia occurs more commonly in patients with eclampsia $(30 \%)$ than in patients with both mild and severe forms of preeclampsia (15-18\%). Patients have severe preeclampsia, with $4-12 \%$ of them having criteria for HELLP syndrome, and immune-mediated thrombocytopenia is responsible for $4.1 \%$ of cases, whereas the other causes such as phospholipid syndrome, drugs, etc. constitute the rest. ${ }^{13}$ It had been concluded that mild-to-moderate thrombocytopenia in pregnancy is not associated with any adverse effects to the neonates or to the mothers, and no active management is necessary other than periodic monitoring.

Some investigators have proposed biochemical markers to predict the severity of hypertensive disorders of pregnancy like Placental tissue protein 13 and Endoglin's; but these tests cannot be used for simple, low-cost screening. Therefore, there is a need to identify a simple test specifically designed for routine use in a hospital environment in particular those suitable at a rural setup. ${ }^{9}$

This study was done to estimate the incidence of thrombocytopenia in pregnant women diagnosed with hypertensive disorders and to correlate the severity of it with the degree of thrombocytopenia.

Thrombocytopenia occurs more commonly in patients with eclampsia (30\%) than in patients with both mild and severe forms of preeclampsia (15-18\%). Patients have severe preeclampsia, with $4-12 \%$ of them having criteria for HELLP syndrome, and immune-mediated thrombocytopenia is responsible for $4.1 \%$ of cases, whereas the other causes such as phospholipid syndrome, drugs, etc. constitute the rest. ${ }^{21}$ It had been concluded that mild-to-moderate thrombocytopenia in pregnancy is not associated with any adverse effects to the neonates or to 
the mothers, and no active management is necessary other than periodic monitoring.

Some investigators have proposed biochemical markers to predict the severity of hypertensive disorders of pregnancy like Placental tissue protein 13 and Endoglin's; but these tests cannot be used for simple, low-cost screening. Therefore, there is a need to identify a simple test specifically designed for routine use in a hospital environment in particular those suitable at a rural setup. ${ }^{9}$

This study was done to estimate the incidence of thrombocytopenia in pregnant women diagnosed with hypertensive disorders and to correlate the severity of it with the degree of thrombocytopenia.

\section{METHODS}

This study was carried out following approval by the Institutional Ethics Committee on 150 pregnant women who had been admitted to the labor and maternity wards of Rajarajeswari Medical College and Hospital, Bengaluru with a diagnosis of hypertensive disorders of pregnancy, during August 2015-August 2016.

Relevant data were obtained from case files and compiled by a common proforma that included socio-demographic characteristics of mothers, obstetric history, signs and symptoms in mother at presentation, laboratory data, and maternal and perinatal outcomes. The data collection was followed by analysis of the collected data.

Any illness in the mother like chronic HTN or illness likely to cause changes in the platelet counts like connective tissue diseases, ITP, Leukaemia, lymphoproliferative diseases and those who received drugs like aspirin were excluded from the study.

Only cases where the blood pressure (BP) and platelet counts that were seen to be consistently abnormal even on retesting following an interval of $6 \mathrm{~h}$ and no more than
1 week apart, as observed in the case files were included in the study.

Based on the criteria described by the National High BP Education Working Group in 2000, the PIH cases in our study were classified into five groups as described by Wolde et al into ${ }^{10}$

\section{- Gestational HTN}

- Mild preeclampsia

- Severe preeclampsia

- Eclampsia

- Haemolysis, elevated liver enzyme levels, and low platelet levels (HELLP) syndrome

Thrombocytopenia was classified as mild when platelet count was found to be 1-1.5 lakh/cumm, moderate at 50,000-1 lakh/cumm and severe with $<50000 /$ cumm. $^{11}$

Platelet counts had been estimated in EDTA anticoagulated blood by SYSMEX KX21 haematology 3 part analyser. In patients with very low platelet count, the counts had been rechecked by peripheral smear examination.

\section{Statistical analysis}

The patient files were analysed to look for any maternal or neonatal complications. Data were arranged in Microsoft Excel version 2010, and statistically analysed by SPSS version 23 .

\section{RESULTS}

The present study included 150 cases of hypertensive disorders of pregnancy, of which $2.6 \%$ (4) were diagnosed with gestational HTN, 56\%(84) with mild preeclampsia, $36 \%(54)$ severe preeclampsia, and 5.3\%(8) had eclampsia. 10 out of 54 cases of severe preeclampsia had features of HELLP syndrome. The cases ranged from 18 to 32 years of age with a mean age of 22 years (Table 1).

Table 1: Platelet count and hypertensive disorders of pregnancy.

\begin{tabular}{|c|c|c|c|c|c|c|c|c|}
\hline \multirow{3}{*}{$\begin{array}{l}\text { Type of hypertensive } \\
\text { disorders of pregnancy } \\
\text { (total number of cases) }\end{array}$} & \multicolumn{6}{|c|}{ Thrombocytopenia } & \multicolumn{2}{|c|}{$\begin{array}{l}\text { Normal platelet } \\
\text { count }\end{array}$} \\
\hline & Mild & & Mod & & Severe & & & \\
\hline & No. of cases & $\%$ & No. of cases & $\%$ & No. of cases & $\%$ & No. of cases & $\%$ \\
\hline Gestational HTN (4) & 2 & 50 & 0 & - & 0 & - & 2 & 50 \\
\hline Mild pre-eclampsia (84) & 44 & 52.3 & 16 & 19.2 & 4 & 4.7 & 20 & 23.8 \\
\hline Severe pre-eclampsia (44) & 18 & 40.9 & 22 & 50 & 0 & - & 4 & 9.09 \\
\hline $\begin{array}{l}\text { Severe pre-eclampsia with } \\
\text { HELLP syndrome (10) }\end{array}$ & 2 & 20 & 6 & 60 & 2 & 20 & 0 & - \\
\hline Eclampsia (8) & 0 & - & 2 & 25 & 2 & 25 & 4 & 50 \\
\hline Total (150) & 66 & 4 & 46 & 30.6 & 8 & 5.3 & 30 & 20 \\
\hline
\end{tabular}


Of the 150 PIH cases, 30 cases showed normal platelet count and 120 cases showed deranged platelet count. Mild thrombocytopenia was seen in $44 \%$, moderate thrombocytopenia was seen in $30.6 \%$ and severe thrombocytopenia was seen in $5.3 \%$.

In the 4 cases of gestational HTN, 2 cases (50\%) had mild thrombocytopenia while the other 2 had a normal platelet count. In the 84 cases of mild preeclampsia, 64 cases $(76.2 \%)$ had thrombocytopenia while the rest 20 $(23.8 \%)$ had normal platelet counts.

Of these 84 cases, 44 cases $(52.4 \%)$ showed mild thrombocytopenia, $16 \quad(19 \%)$ showed moderate thrombocytopenia, and $4(4.7 \%)$ cases had severe thrombocytopenia.
In the 44 cases of severe preeclampsia, 40 cases $(90.9 \%)$ had thrombocytopenia while the rest $4(9.09 \%)$ had normal platelet counts. Of these 40 cases, 18 cases $(40.9 \%)$ showed mild thrombocytopenia, 22 (50\%) showed moderate thrombocytopenia, and none had severe thrombocytopenia. 10 cases of severe preeclampsia manifested with findings of HELLP syndrome and all had thrombocytopenia. Of these 10 cases 2 cases (20\%) showed mild thrombocytopenia, 6 (60\%) showed moderate thrombocytopenia, and $2(20 \%)$ had severe thrombocytopenia. In the 8 cases of eclampsia, 4 cases $(50 \%)$ had thrombocytopenia while the rest $4(50 \%)$ had normal platelet counts. Of these 4 cases, 2 cases each showed moderate, and severe thrombocytopenia (Table 1).

Table 2: Distribution of cases based on parity and gravida status.

\begin{tabular}{|llllllllll|}
\hline $\begin{array}{l}\text { Type of hypertensive } \\
\text { disorders of pregnancy }\end{array}$ & Parity & \multicolumn{9}{c}{ Gravida } & \multicolumn{2}{c}{ Total } \\
\hline & Primi & Multi & Total & $\mathbf{1}$ & $\mathbf{2}$ & $\mathbf{3}$ & $\mathbf{4}$ & $\mathbf{5}$ \\
\hline Gestational HTN & 0 & 4 & 4 & 0 & 4 & Nil & Nil & Nil & 4 \\
\hline Mild pre-eclampsia & 52 & 32 & 84 & 52 & 16 & 12 & 4 & Nil & 84 \\
\hline Severe pre-eclampsia & 34 & 10 & 44 & 34 & 8 & 2 & Nil & Nil & 44 \\
\hline $\begin{array}{l}\text { Severe pre-eclampsia with } \\
\text { HELLP syndrome }\end{array}$ & 6 & 4 & 10 & 6 & Nil & 4 & Nil & Nil & 10 \\
\hline Eclampsia & 6 & 2 & 8 & 6 & Nil & 2 & Nil & Nil & 8 \\
\hline Total cases & 98 & 52 & 150 & 98 & 28 & 20 & 4 & Nil & 150 \\
\hline
\end{tabular}

Table 3: Comparison of maternal and fetal outcomes with severity of PIH.

\begin{tabular}{|llll|}
\hline Type of PIH & Number of cases & Maternal complications (No.) & Foetal complications (No.) \\
\hline Gestational HTN & 4 & - & - \\
\hline Mild pre-eclampsia & 84 & - & IUGR (8) \\
\hline Severe pre-eclampsia & 54 & $\begin{array}{l}\text { HELLP (10) } \\
\text { PPH (0) } \\
\text { Abruption (4) }\end{array}$ & IUGR (14) \\
Eclampsia & 8 & $\begin{array}{l}\text { PPH (3) } \\
\text { DIC with maternal death (0) }\end{array}$ & IUFD (2) \\
\hline Total cases & 150 & 17 & 27 \\
\hline
\end{tabular}

Case of preeclampsia, eclampsia, and HELLP syndrome were found to be more common in the primigravida patients $(67 \%)$, while gestational HTN was seen only in multiparous patients (Table 2).

In the various types of $\mathrm{PIH}, 11 \%$ of cases showed poor maternal outcome and $18 \%$ cases showed poor foetal outcome. Maternal complications were only seen in eclampsia (3/8 cases, 37.5\%) and severe preeclampsia cases (14/54 cases, $25.9 \%)$. Of maternal complications there were 3 cases $(37.5 \%)$ with postpartum hemorrhage $(\mathrm{PPH})$ in the 8 cases of eclampsia. 10 cases of severe preeclampsia had features of HELLP syndrome $(18.5 \%)$ and 4 case had abruption (7.4\%). There were no maternal complications observed in the gestational and mild preeclampsia cases. An incidence of $18 \%$ foetal complications were observed in which perinatal mortality was seen in 2 cases belonging to eclampsia group and in 4 cases of severe pre-eclampsia followed by 14 cases and 8 cases of intrauterine growth restriction (IUGR) in the severe and mild preeclampsia group. None of the cases with gestational HTN showed any foetal complications.

\section{DISCUSSION}

One of the most common medical complications of pregnancy is $\mathrm{PIH}$; generally more common in the developing countries than in the developed countries. ${ }^{10,12}$ 
Risk factors like extreme age, nulliparity, and race have been documented to be associated with this condition by various studies. ${ }^{10}$ There is increasing evidence of the pivotal role of platelets in the development of preeclampsia and intra-uterine growth retardation. ${ }^{22,23}$

The thrombocytopenia in pregnancy-induced hypertension is moderate, and the platelets rarely drop below 20,000/L. ${ }^{24}$ Hemorrhage is an uncommon event unless the patient develops disseminated intravascular coagulation, but thrombocytopenia can be a sign of worsening hypertensive disease. A slowly decreasing platelet count can be noted before the clinical manifestations of pregnancy induced hypertension. When accompanied by microangiopathic hemolytic anemia, hemolysis, elevated liver enzymes, and low platelets, HELLP syndrome is diagnosed. The causes of thrombocytopenia from pregnancy-induced hypertension and HELLP syndrome are unknown. One explanation is that it might be related to abnormal vascular tone with resultant accelerated platelet destruction, platelet activation, and coagulation defects. ${ }^{24}$ The increased levels of platelet-associated immunoglobulin $\mathrm{G}(\mathrm{IgG})$ that have been detected in patients with pregnancy-induced hypertension have been shown to be nonspecific and do not necessarily imply an immunologic basis for the thrombocytopenia. ${ }^{24}$

The treatment for progressive pre-eclampsia, including thrombocytopenia, remains delivery, and the thrombocytopenia then rapidly resolves. ${ }^{25}$ The ongoing CLASP trial should determine whether low-dose aspirin can prevent pre-eclampsia, which would represent a major advance in managing this problem. The HELLP syndrome (haemolysis, elevated liver function tests, low platelet counts) is a serious complication of preeclampsia, affecting about $8.5 \%$ of severe pre-eclamptics, and perinatal mortality may approach $50 \% .{ }^{26,27}$ It is vital to recognize the syndrome, even in the absence of marked hypertension, as recovery may necessitate immediate delivery, despite fetal immaturity. ${ }^{26,28}$ Following delivery, the illness regresses rapidly. Close monitoring of a stable patient may allow fetal maturation. ${ }^{26}$ Infants of preeclamptic women are unlikely to be thrombocytopenic, except when they have other complications of prematurity. ${ }^{29,30}$

In a study from Ethiopia $66.7 \%$ of cases with PIH studied by Wolde et al were nulliparous that is similar to present study where $63 \%$ of cases were primigravida and studies by Riaz where $60 \%$ of the cases were primigravida. ${ }^{31,32}$ More severe forms of PIH were found to be more common in nulliparous ladies as seen in our study. The preponderance of primigravida cases accounts for the young age group of the present study group. Similar findings have been observed by Vinodhini et al (24-29 years) and Meshram et al (mean age of patients with preeclampsia 24.55 years and eclampsia 24.30 years). ${ }^{5,33}$ Annually around 1 lakh women die worldwide due to eclampsia with an estimated rate of one death every 3 min worldwide caused by preeclampsia and eclampsia. Preeclampsia causes poor maternal and fetal outcomes having a predictable onset and progression that may be cured by termination of pregnancy. ${ }^{5}$ In India, PIH continues to be responsible for the largest proportion of perinatal deaths resulting from prematurity and IUGR; and is a major contributor to perinatal mortality. Though most of these conditions can be prevented, or identified and treated early by good antenatal care; the situation is still not very bright in rural India. ${ }^{5}$

Various systems of classifications are noted in literature with modifications being made constantly making comparison difficult. ${ }^{33}$ Bangal et al excluded gestational HTN and clubbed mild and severe PIH. ${ }^{34}$ While Mohammed et al have studied the variations of platelet indices in PIH in a fixed study group of 20 cases each under each category, whereby the incidence of each cannot be commented upon and Parmar et al have categorized PIH cases into mild, moderate and severe $\mathrm{PIH}^{35,36}$ Wolde et al's method to categorize cases with PIH based on the National High BP Education Working Group (2000) criteria has been done in this study owing to feasibility and simplicity of categorization into the five mentioned groups. ${ }^{31}$

In a study from Bhopal by Anand and Kirshnanand et al majority of the cases had preeclampsia $(66.36 \%)$ and the rest eclampsia $(33.64 \%){ }^{3}$ Wolde et al's study showed preeclampsia as the most common hypertensive disorder of pregnancy (51.9\%); followed by eclampsia (23.4\%), HELLP syndrome $(8.9 \%)$, mild preeclampsia $(7.6 \%)$, and simple gestational HTN $(5.1 \%) .{ }^{31}$ In present study, most cases belonged to the mild preeclampsia (56\%) group followed by cases with severe preeclampsia (30\%). The incidence of eclampsia however was much lower in our study $(6 \%)$. These differences could be due to small sample size of our study and the social ethnic and cultural difference in group of population studied as noted by Wolde et al who has quoted black race as one of the risk factors for $\mathrm{PIH}{ }^{31}$

It has been shown by earlier researchers that hematological aberrations such as thrombocytopenia and reduction in some plasma clotting factors may develop in preeclampsia women. ${ }^{37,38}$ A transient mild thrombocytopenia is seen due to increased platelet consumption during pregnancy. Thrombocytopenia is found in approximately $6 \%$ of pregnancies and most common cause of thrombocytopenia in pregnancy is preeclampsia and eclampsia. A continuous decline in platelet count as pregnancy advances was shown by Fay et al and Shah AR et al indicated that there is possibility of platelet hyper destruction during pregnancy. ${ }^{39,40}$ This together with hemodilution and platelet trapping results in decreased platelet. $^{8}$ McCrae has suggested that thrombocytopenia may precede the various other manifestations of preeclampsia, and thus should be considered in the event of isolated thrombocytopenia seen in the late second or third trimester. 
Some authors have suggested that thrombocytopenia is caused due to peripheral consumption, endothelial damage, and reduced life span. Altered platelet membrane with accelerated aggregation and destruction have also been suggested. ${ }^{8}$

Meshram et al in a hospital based study found lower platelet counts in preeclampsia and eclampsia that in healthy pregnant controls. ${ }^{5}$ Low platelet count was seen in $29.31 \%$ of cases with preeclampsia and in $44.44 \%$ of cases with eclampsia. Studies by Khan A et al from Pakistan found a fall in platelet counts in cases with PIH. The authors in their study have documented similar findings from India by Annam $\mathrm{V}$ et al and Turkey by Yaprak EU et al.

In present study though thrombocytopenia was a consistent finding, a systematic fall in platelet counts in relation to the severity of $\mathrm{PIH}$ however was not noted, with $50 \%$ in cases with milder form of disease i.e. gestational HTN and $50 \%$ in severe form of disease i.e. eclampsia, showing normal platelet counts. Majority of cases with preeclampsia had thrombocytopenia and all cases complicated with HELLP syndrome had thrombocytopenia. Hence though thrombocytopenia may help in identifying progression of disease, but its absence does not rule out a severe disease. Hence, an overall consideration of various laboratory tests than just one test has been made obvious.

In the present study, the incidence of HELLP syndrome was $6 \%$, the values of present study are consistent with that of Riaz et al who reported an incidence of $5 \% .^{32}$ Meshram et al reported an incidence of $10.63 \%$ and in their study documented similar findings by Sibai et al $9.8 \%$ and $\mathrm{J}$ Prakash et al findings of $2-12 \% .^{5}$ Studies by Odegard et al have shown pregnancies complicated by severe preeclampsia to have infants born with low birth weights; being around $12 \%$ below than expected, while pregnancies with mild preeclampsia showing no significant difference from expected range.

The "Born Too Soon" report by Raina and Mehta provides estimates for global comparisons. According to this report, annually 15 million babies are born before 37 weeks gestation with one of the causes being PIH. In present study the incidence of IUGR in preeclamptic patients was found to be $16 \%$ which was comparable to Ludec et al $(21 \%)$ and Samantha et al $(15.5 \%)$ and Meshram et al $(19.14 \%) .^{5}$

The incidence of PPH as a complication in PIH was found to be $2 \%$ in present study. The incidence rate is lesser than those observed by Meshram et al $(8.5 \%)$ and Ludec et al (10.20\%). These differences could stem from the variations in therapeutic strategies at an individual level down to an Institutional level and to the possible genetic factors that may have a role in affecting progression of disease or help in an adaptive response preventing complications.

\section{CONCLUSION}

Platelet count is a simple, low cost, and rapid routine screening test. Varied conclusions have been drawn linking platelet count to severity of PIH. As a normal count does not rule out a severe disease our study shows that platelet count alone cannot be relied upon to assess the severity of PIH. Uniformity in utilization of classification and categorization of cases with PIH is also needed for better understanding of the disease process. The search for one marker that would identify and gauge the severity of PIH still continues.

Pregnancy induced thrombocytopenia is not a major complication of pregnancy even in hypertensive pregnant women during the third trimester; however, postpartum and during subsequent pregnancies, follow up by obstetrician, physician, and paediatrician is recommended.

Funding: No funding sources

Conflict of interest: None declared

Ethical approval: The study was approved by the Institutional Ethics Committee

\section{REFERENCES}

1. Mohapatra S, Pradhan BB, Satpathy UK, Mohanty A, Pattnaik JR. Platelet estimation: Its prognostic value in pregnancy induced hypertension. Indian J Physiol Pharmacol. 2007;51:160-4.

2. Sivakumar S, Bhat BV, Badhe BA. Effect of pregnancy induced hypertension on mothers and their babies. Indian J Pediatr. 2007;74:623-5.

3. Anand S, Kirshnanand. Perinatal outcome in growth retarded babies born to normotensive and hypertensive mothers: A prospective study. People's J Sci Res. 2012;5:24-8.

4. Agarwal S, Buradkar S. Coagulation studies in toxemia of pregnancy. J Obstet Gynaecol India. 1978;27:992-6.

5. Meshram DP, Chavan YH, Kadam PN, Panchal MG, Ramteke DJ. Maternal and foetal outcomes in pregnancy induced hypertension: A hospital based study. Int J Pharm Sci Invention. 2014;3:23-6.

6. Leduc L, Wheeler JM, Kirshon B, Mitchell P, Cotton DB. Coagulation profile in severe preeclampsia. Obstet Gynecol. 1992;79:14-8.

7. Chauhan P, Rawat U, Bisht V, Purohit RC. Comparison of coagulation profile in pre-eclamptic and eclamptic patients with normotensive pregnant patients. J Evol Med Dent Sci. 2014;3:3208-15.

8. Veena HC, Manjunatha S, Itagi V, Taklikar RH, Patil RS. The hemostatic mechanisms in PIH. Indian J Appl Basic Med Sci. 2015;17:40-4.

9. Matthews JH, Benjamin S, Gill DS, Smith NA. Pregnancy-associated thrombocytopenia: Definition, incidence and natural history. Acta Haematol. 1990;84:24.

10. Boehlen F, Hohlfeld P, Extermann P, Perneger TV, De Moerloose P. Platelet count at term pregnancy: a reappraisal of the threshold. Obstet Gynecol. 2000;95:29-33. 
11. George JN, Woolf SH, Raskob GE, Wasser JS, Aledort LM, Ballem PJ et al. Idiopathic thrombocytopenic purpura: A practice guideline developed by explicit methods for the American society of haematology. Blood. 1996;88:3-10.

12. Burrows RF, Kelton JG. Thrombocytopenia at delivery: A prospective survey of 6175 deliveries. Am J Obstet Gynecol. 1990;162:731-4.

13. Burrows RF, Kelton JG. Fetal thrombocytopenia and its relation to maternal thrombocytopenia. $\mathrm{N}$ Engl J Med. 1993;329:1463.

14. Kaplan C, Forestier F, Dreyfus M, Morel-Kopp MC, Tchernia G. Maternal thrombocytopenia during pregnancy: Diagnosis and etiology. Semin Thromb Hemost. 1995;21:85.

15. Tsunoda $\mathrm{T}$, Ohkuchi A, Izumi A, Watanabe $\mathrm{T}$, Matsubara S, Sato I, et al. Antithrombin III activity and platelet count are more likely to decrease in twin pregnancies than in singleton pregnancies. Acta Obstet Gynecol Scand. 2002;81:840.

16. Letsky EA, Greaves M. Guidelines on the investigation and management of thrombocytopenia in pregnancy and neonatal alloimmune thrombocytopenia. Br J Haematol. 1996;95:21.

17. Moise KJ. Autoimmune thrombocytopenic purpura in pregnancy. Clin Obstet Gynecol. 1991;34:51.

18. Burrows RF, Kelton JG. Pregnancy in patients with idiopathic thrombocytopenic purpura: Assessing the risks for the infant at delivery. Obstet Gynecol Surv. 1993;48:781.

19. Saftlas AF, Olson DR, Franks AL, Atrash HK, Pokras R. Epidemiology of preeclampsia and eclampsia in the United States, 1979-1986. Am J Obstet Gynecol. 1990; $163: 460$.

20. Sibai BM, Ramadan MK, Chri RS, Friedman SA. Pregnancies complicated by HELLP syndrome (hemolysis, elevated liver enzymes, and low platelets): Subsequent pregnancy outcome and long-term prognosis. Am J Obstet Gynecol. 1995;172:125.

21. Magann EF, Martin JN Jr. Twelve steps to optimal management of HELLP syndrome. Clin Obstet Gynecol. 1999;42:532-50.

22. Zemel MB, Zemel PC, Berry S, Norman G, Kowalczyk $\mathrm{C}$, Sokol RJ et al. Altered platelet calcium metabolism as an early predictor of increased peripheral vascular resistance and pre-eclampsia in urban black women. $\mathrm{N}$ Engl J Med. 1990;323:434-7.

23. Redman CWG. Platelets and the beginnings of preeclampsia (editorial). N Engl J Med. 1990;323:47880.

24. Katz VL, Thorp JM, Rozas L, Bowes WA. The natural history of thrombocytopenia associated with preeclampsia. Am J Obstet Gynecol. 1990;163:1142-3.

25. Silver R, Berkowitz R, Bussel J. Thrombocytopenia in pregnancy. Practice bulletin, No 6. Chicago: Am Coll Obstet Gynecol. 1999.

26. Weinstein L. Syndrome of hemolysis, elevated liver enzymes, and low platelet count: a severe consequence of hypertension in pregnancy. Am J Obstet Gynecol. 1982;142:159-67.

27. Sibai BM, Spinnato JA, Watson DL, Hill GA, Anderson GD. Pregnancy outcome in 303 cases with severe preeclampsia. Obstet Gynecol. 1984 Sep;64(3):319-25.

28. Weinstein L. Preeclampsia/eclampsia with hemolysis, elevated liver enzymes, and thrombocytopenia. Obstet Gynecol. 1985;66:657-60.

29. Pritchard JA, Cunningham FG, Pritchard SA, Mason RA. How often does maternal preeclampsia-eclampsia incite thrombocytopenia in the fetus?. Obstet Gynecol. 1987 Mar;69(3 Pt 1):292-5.

30. Burrows RF, Andrew M. Neonatal thrombocytopenia in the hypertensive disorders of pregnancy. Obstet Gynecol. 1990;76:234-48.

31. Wolde Z, Segni H, Woldie M. Hypertensive disorders of pregnancy in Jimma University specialized hospital. Ethiop J Health Sci. 2011;21:147-54.

32. Riaz S, Habib S, Jabeen A. Frequency of maternal mortality and morbidity in pregnancy-induced hypertension. J Ayub Med Coll Abbottabad. 2011;23:61-3.

33. Vinodhini R, Kumari L. Evaluation of platelet count as a prognostic index in eclampsia and pre eclampsia. Int $\mathrm{J}$ Mod Res Rev. 2014;2(10):447-52.

34. Bangal VB, Giri PA, Mahajan AS. Maternal and foetal outcome in pregnancy induced hypertension: A study from rural tertiary care teaching hospital in India. Int $\mathbf{J}$ Biomed Res. 2011;2:595-9.

35. Mohammed FE, Khalil HB, Idriss MI, Abdalla TE, Elbadawi NE. Variations of platelets indices in pregnancy induced hypertension. J Physiobiochem Metab. 2013;2:1.

36. Parmar MT, Solanki HM, Gosalia VV. Study of risk factors of perinatal death in pregnancy induced hypertension (PIH). Natl J Community Med. 2012;3:703-7.

37. Sameer MA, Meshram DP, Deshpande SA, Sadhu D, Pandit S. Role of platelet count as important prognostic marker in pregnancy induced hypertension. IOSR J Dent Med Sci. 2014;13:39-43.

38. Mohapatra D, Priyadarsini N, Behera M, Panda P, Mishra T. Hematological parameters in the assessment of pregnancy induced hypertension. Int $\mathrm{J}$ Pharm Bio Sci. 2015;6:854-9.

39. Fay RA, Hughes AO, Farron NT. Platelets in pregnancy: Hyper destruction in pregnancy. Obstet Gynecol. 1983;61:238.

40. Shah AR, Chaudhari SN, Shah MH. Role of platelet parameters in diagnosing various clinical conditions. Natl J Med Res. 2013;3:162-5.

Cite this article as: Rupakala BM, Gupta R, Shetty $\mathrm{VH}$, Sailakshmi MPA. Thrombocytopenia in hypertensive disorders of pregnancy. Int J Reprod Contracept Obstet Gynecol 2018;7:848-54. 